\section{Spontaneous disintegration of a retained video capsule in a patient with cryptogenic multifocal ulcerous stenosing enteritis: a rare complication}

A 36-year-old woman with abdominal pain, diarrhea, and weight loss underwent capsule endoscopy after a normal enteroclysis. The capsule study revealed small-bowel ulcers and erosions and a diagnosis of cryptogenic multifocal ulcerous stenosing enteritis was made $[1,2]$ (๑ Fig. 1, 2). Enteropathy due to nonsteroidal anti-inflammatory use, Crohn's disease, and celiac disease were all ruled out. Asymptomatic capsule retention in the ileum was diagnosed subsequently ( Fig. 3). The patient was not fit for surgery. She died after 7 months as a result of bilateral bronchopneumonia complicated by sepsis. At autopsy, six short stenosing strictures and a video capsule disintegrated into three parts were discovered in the distal ileum ( Fig. 4). Histological examination revealed superficial erosions and reparative changes with granulation tissue and scar formation in the mucosa and submucosa ( $\bullet$ Fig. 5).

The longest reported duration of video capsule retention is 2.5 years and the International Conference on Capsule En-

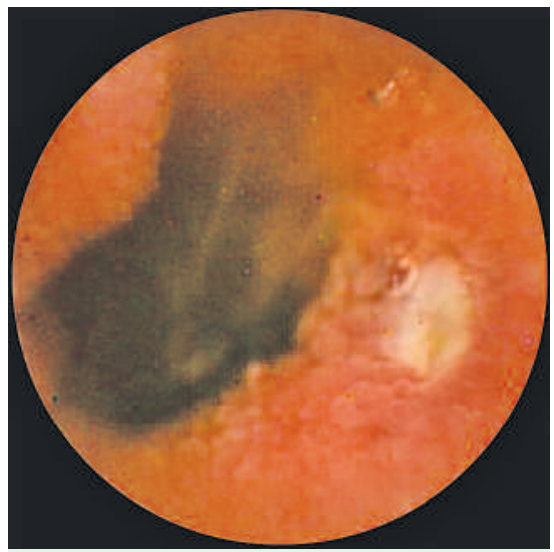

Fig. 1 Capsule endoscopy image from the proximal ileum showing a small, roundish ulcer with a fibrin base and mild inflammation nearby. doscopy (ICCE) consensus for capsule retention stated no time limit for removing a retained capsule [3]. Only one case of capsule disintegration (after retention in the ileum for 6 months) has been reported so far [4].

To the best of our knowledge, this is only the second reported case of video capsule retention (caused by multiple ileal stenoses) resulting in disintegration of the capsule. The mechanism of capsule disintegration is not clear but the combination of chronic exposure to digestive and bacterial enzyme activity and peristalsis on the joint between the optical dome and the capsule body is the most probable explanation. There are some potential problems in this situation. The smaller components of the disintegrated video capsule could be caught within the stricture and induce ileus, and the sharp parts could injure the intestinal wall. On the other hand, the capsule's batteries are only minimally toxic, containing silver oxide.

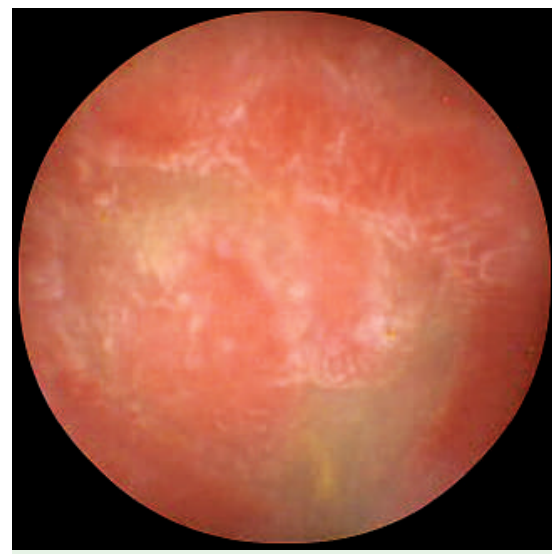

Fig. 2 A capsule endoscopy image showing a stellar ulcer in the proximal jejunum.
In reporting this case we want to draw attention to the possible risk of capsule disintegration after a long period of retention. The length of time which an asymptomatic retained capsule can be safely left in the small bowel should therefore be determined.

\section{Acknowledgment \\ $\nabla$}

This study was supported by research project MZO 00179906 from the Ministry of Health of the Czech Republic.

Endoscopy_UCTN_Code_CPL_1AI_2AB

I. Tachecí ${ }^{1}$, A. Ryška², S. Rejchrt ${ }^{1}$, M. Kopáčová ${ }^{1}$, V. Hořava ${ }^{3}$, J. Bureš Second Department of Internal Medicine, Charles University Faculty of Medicine and Teaching Hospital, Hradec Králové, Czech Republic

2 The Fingerland Department of Pathology, Charles University Faculty of Medicine and Teaching Hospital, Hradec Králové, Czech Republic

3 Department of Pathology, Municipal Hospital, Ostrava, Czech Republic

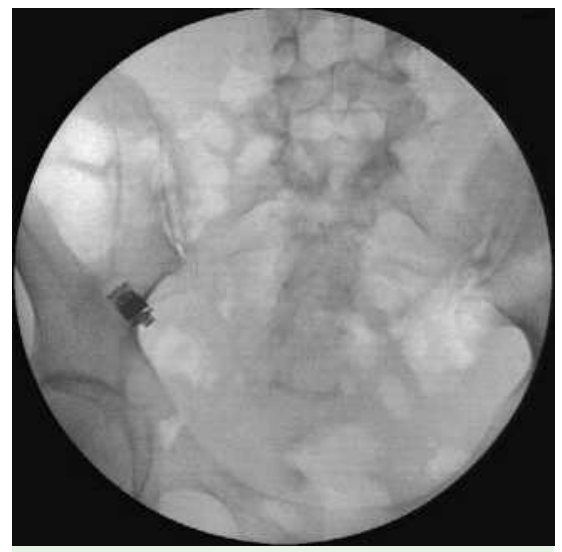

Fig. 3 Fluroscopy performed after the capsule endoscopy confirmed that the capsule was still in the ileum. 


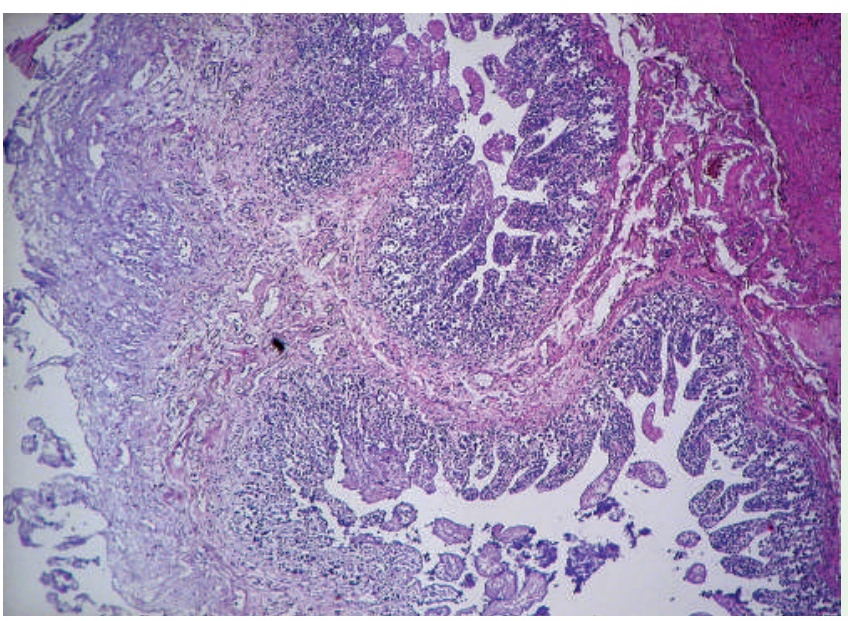

Fig. 5 Histological findings included focal mucosal fibrosis with proliferation of granulation tissue. The epithelium was desquamated as a result of postmortem autolytic changes (hematoxylin and eosin stain, original magnification $\times 200$ ).

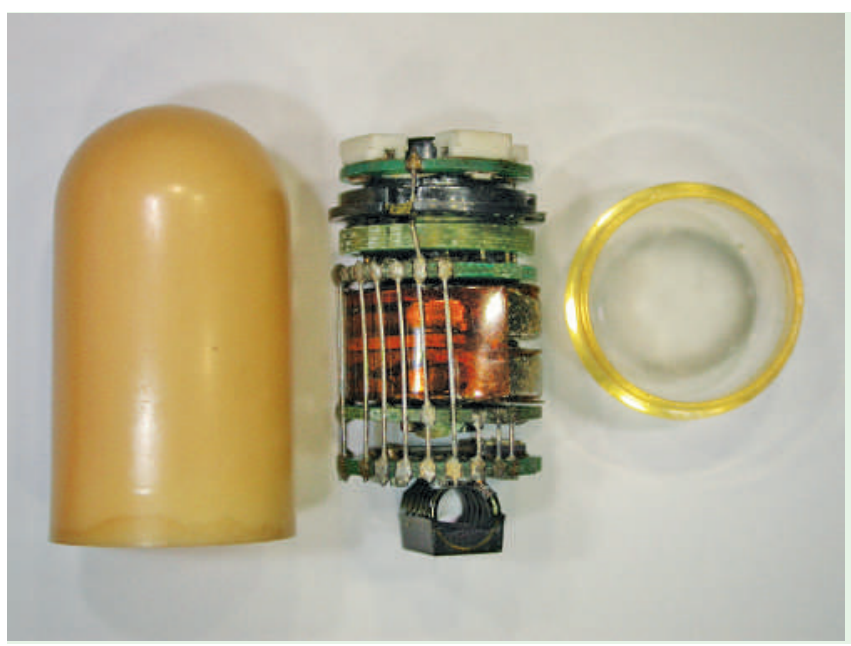

Fig. 4 The capsule disintegrated into three parts (the optical dome, the plastic body, and the capsule's internal components) after its removal from the ileum at autopsy.

\section{References}

1 Perlemuter G, Guillevin L, Legman P et al. Cryptogenetic multifocal ulcerous stenosing enteritis: an atypical type of vasculitis or a disease mimicking vasculitis. Gut 2001; 48: 333-338

2 Matsumoto T, Iida M, Matsui T et al. Nonspecific multiple ulcers of the small intestine unrelated to non-steroidal anti-inflammatory drugs. J Clin Pathol 2004; 57: 1145 1150

3 Cave D, Legnani $P$, de Franchis $R$ et al. ICCE consensus for capsule retention. Endoscopy 2005; 37: $1065-1067$

4 Fry LC, De Petris G, Swain JM et al. Impaction and fracture of a video capsule in the small bowel requiring laparotomy for removal of the capsule fragments. Endoscopy 2005; 37: $674-676$

Bibliography

DOI 10.1055/s-2007-966871

Endoscopy 2008; 40: E104-E105

(c) Georg Thieme Verlag KG Stuttgart · New York . ISSN 0013-726X

\section{Corresponding author}

\section{Tacheci, MD}

Second Department of Internal Medicine Faculty of Medicine at Hradec Králové Charles University Teaching Hospital Sokolska 581

Hradec Králové 50011

Czech Republic

Fax: +420-5832003

tacheci@gmail.com 